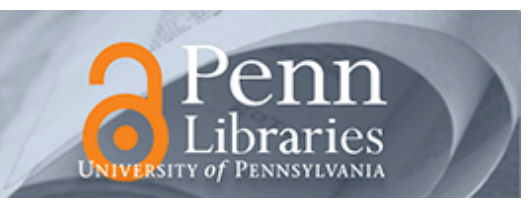

University of Pennsylvania

ScholarlyCommons

\title{
Calculation of the Positions of the $\boldsymbol{\alpha}$ - and $\boldsymbol{\beta}$-bands in the Electronic Spectra of Benzenoid Hydrocarbons Using the Method of Limited Configuration Interaction
}

Josef Koutecký

Josef Paldus

Vaclav Vitek

University of Pennsylvania, vitek@seas.upenn.edu

Follow this and additional works at: https://repository.upenn.edu/mse_papers

Part of the Atomic, Molecular and Optical Physics Commons, Biochemical and Biomolecular Engineering Commons, and the Semiconductor and Optical Materials Commons

\section{Recommended Citation}

Koutecký, J., Paldus, J., \& Vitek, V. (1963). Calculation of the Positions of the $\alpha$ - and $\beta$-bands in the Electronic Spectra of Benzenoid Hydrocarbons Using the Method of Limited Configuration Interaction. Collection of Czechoslovak Chemical Communications, 28 (6), 1468-1482. http://dx.doi.org/10.1135/ cccc19631468

At the time of publication, author Vaclav Vitek was affiliated with the Czechoslovak Academy of Sciences. Currently, he is a faculty member at the Materials Science and Engineering Department in the School of Engineering and Applied Science at the University of Pennsylvania.

This paper is posted at ScholarlyCommons. https://repository.upenn.edu/mse_papers/226

For more information, please contact repository@pobox.upenn.edu. 


\title{
Calculation of the Positions of the $\boldsymbol{\alpha}$ - and $\boldsymbol{\beta}$-bands in the Electronic Spectra of Benzenoid Hydrocarbons Using the Method of Limited Configuration Interaction
}

\author{
Abstract \\ The positions of the $\alpha$ - and $\beta$-bands in the electronic absorption spectra of twenty aromatic benzenoid \\ hydrocarbons were calculated by the semiempirical method of limited configuration interaction in the $\pi$ - \\ electron approximation using the Huckel molecular orbitals. The agreement of the experimental and \\ calculated values is good for the $\beta$-band whereas a systematic deviation is observed for the $\alpha$-band. This \\ deviation cannot be removed by extending the configuration interaction of the monoexcited states \\ constructed from the molecular orbitals considered. However, the consideration of electronic repulsion \\ enables us to explain the character of the dependences of the experimental excitation energies on the \\ excitation energies obtained by the simple Huckel method of molecular orbitals. Using a suitable choice \\ of semiempirical parameters different for various electronic transitions (showing no large mutual \\ differences) yields semiempirical interpolation formulas for the; $p$-, $\alpha$-, and $\beta$-bands which give very good \\ agreement with the corresponding experimental excitation energies for the compounds studied.

\section{Disciplines} \\ Atomic, Molecular and Optical Physics | Biochemical and Biomolecular Engineering | Engineering | \\ Materials Science and Engineering | Semiconductor and Optical Materials

\section{Comments} \\ At the time of publication, author Vaclav Vitek was affiliated with the Czechoslovak Academy of Sciences. \\ Currently, he is a faculty member at the Materials Science and Engineering Department in the School of \\ Engineering and Applied Science at the University of Pennsylvania.
}




\title{
CALCULATION OF THE POSITIONS OF THE $\alpha$ - AND $\beta$-BANDS IN THE ELECTRONIC SPECTRA OF BENZENOID HYDROCARBONS USING THE METHOD OF LIMITED CONFIGURATION INTERACTION
}

\author{
J. KouteckÝ, J. Paldus and V. VÍteK \\ Institute of Physical Chemistry, Czechoslovak Academy of Sciences, Prague, \\ and Mathematical-Physical Faculty of the Charles University, Prague
}

Received April 17th, 1962

\begin{abstract}
The positions of the $\alpha$ - and $\beta$-bands in the electronic absorption spectra of twenty aromatic benzenoid hydrocarbons were calculated by the semiempirical method of limited configuration interaction in the $\pi$-electron approximation using the Hückel molecular orbitals. The agreement of the experimental and calculated values is good for the $\beta$-band whereas a systematic deviation is observed for the $\alpha$-band. This deviation cannot be removed by extending the configuration interaction of the monoexcited states constructed from the molecular orbitals considered. However, the consideration of electronic repulsion enables us to explain the character of the dependences of the experimental excitation energies on the excitation energies obtained by the simple Hückel method of molecular orbitals. Using a suitable choice of semiempirical parameters different for various electronic transitions (showing no large mutual differences) yields semiempirical interpolation formulas for the $p-, \alpha-$, and $\beta$-bands which give very good agreement with the corresponding experimental excitation energies for the compounds studied.
\end{abstract}

In a recent study ${ }^{1}$ the positions of the $p$-bands (according to the Clar classification ${ }^{2}$ ) of twenty benzenoid hydrocarbons have been calculated using the method of limited configuration interaction in the $\pi$-electron approximation. The LCAO molecular orbitals in the Hückel approximation were used for the construction of the wave functions describing individual configurations. The positions of the $\alpha$ - and $\beta$-bands of the same group of compounds are estimated by the same method ${ }^{1}$ in the work presented.

\section{Method of Calculation}

The method of calculation and the approximations employed are those used in the preceding paper $^{1}$ (compare refs ${ }^{3-6}$ ). The distances between neighboring carbon atoms are taken equal to $1.39 \AA$ which, of course, is a rather crude approximation in some cases. The resonance integral between neighboring atomic orbitals is taken equal to $-2.318 \mathrm{eV}\left(\mathrm{see}^{1}\right)$ and the electronic repulsion integrals of the Coulomb type are approximated according to Mataga and Nishimoto ${ }^{7}$

$$
\gamma_{\mu v}=\mathrm{e}^{2} /\left(1 \cdot 328+r_{\mu v}\right) \text {, }
$$


where $r_{\mu v}$ is the distance between the $\mu$-th and $v$-th carbon atom. Otherwise, the approximations introduced by Pariser and $\mathrm{Parr}^{3,4}$ and Pople ${ }^{5,6}$ are used.

The off-diagonal elements of the effective one-electron Hamiltonian $F$ in the Hückel molecular orbital representation were neglected. We considered the monoexcited configurations to which the Hückel excitation energy lower than $1|\beta|$ corresponds.

The extent of the configuration interaction was investigated. Therefore, the excitation energies $\varepsilon_{\mathrm{p}}^{\mathrm{k}}(k=\alpha, \beta)$ which follow from the interaction of the configurations $\left|1,1 \rightarrow 2^{\prime}\right\rangle$ and $\left|1,2 \rightarrow 1^{\prime}\right\rangle$ of the same energy were also calculated (compare refs ${ }^{6-8}$ ). Here and hereforewith we use the designation, in which $\left|1, i \rightarrow j^{\prime}\right\rangle$ denotes the singlet monoexcited configuration formed by the substitution of the $i$ th bonding molecular orbital with the $j$ th virtual orbital in the monodeterminantal ground state wave function. The orbitals are arranged according to the increasing absolute values of the diagonal matrix elements $F_{\mathrm{ii}}$. The bonding orbitals are denoted by $1,2 \ldots N / 2$ and the virtual orbitals by $1^{\prime}, 2^{\prime} \ldots N^{\prime} / 2$, where $N$ is the number of carbon atoms of the hydrocarbon.

In the cases of naphthacene, pentacene, and dibenzo[b,def $]$ chrysene both the ransitions formed by the interaction of $\left|1,1 \rightarrow 2^{\prime}\right\rangle$ and $\left|1,2 \rightarrow 1^{\prime}\right\rangle$ are, however, torbidden. These monoexcited configurations belong to the irreducible representafion $B_{1 g}$ in naphthacene and pentacene $\left(\mathrm{D}_{2 \mathrm{~h}}\right)$ and to the irreducible representation $A_{g}$ tn dibenzo[b,def $]$ chrysene.

Table I

Assignment or Irreducible Representations to Monoexcited States for Various Transitions According to Clar Classification

$\begin{array}{cccccc}\text { Group } & \mathbf{D}_{6 \mathrm{~h}} & \mathbf{D}_{2 \mathrm{~h}} & \mathbf{C}_{2 \mathrm{v}} & \mathbf{C}_{2 \mathrm{~h}} & \mathbf{C}_{1 \mathrm{~h}} \\ \text { Compound } & 1,20 & 2,4,6,9,11,3,5,10,12,14, & 7,15,19 & 8,13 \\ & & 16 & 17,18 & \\ \alpha & B_{2 u} & B_{2 u}{ }_{a} & B_{2} & B_{u} & A^{\prime} \\ \beta & B_{1:} & B_{3 u} & A_{1} & B_{i t}{ }^{b} & A^{\prime} \\ \beta & E_{1 u} & - & - & - & -\end{array}$

${ }^{a}$ Pentacene is an exception as its monoexcited configurations producing the $\alpha$-transition belong to the irreducible representation $B_{1 g}{ }^{b}$ Dibenzo[bdef ]chrysene is an exception as its monoexcited configurations producing the $\alpha$-transition belong to the irreducible representation $A_{g}$.

The full configuration interaction was considered for four compounds: anthracene $\left(D_{2 h}\right)$, chrysene $\left(C_{2 h}\right)$, benzo $[a]$ anthracene $\left(C_{1 h}\right)$ and benzo $[e]$ pyrene $\left(C_{2 v}\right)$. The configuration interaction for coronene $\left(D_{6 h}\right)$ was extended so that it included the configurations formed by excitations from the nine highest bonding molecular orbitals to the nine lowest virtual orbitals. The consideration of the configurations with the Hückel excitation energy up to $1|\beta|$ for benzene and naphthalene includes all monoexcited configurations. As the off-diagonal elements of the Hamiltonian in the repre- 
sentation of the monoexcited configurations are small for the singlet states in comparison to the diagonal elements the perturbation method may be used for the calculation of the excitation energies of the monoexcited states following from configuration interaction.

If we define the longest-wavelength permitted transition as the $p$-band and the second longest-wavelength permitted transition as the $\beta$-band we obtain for various symmetry the assignment of the corresponding monoexcited states to the irreducible representations as given in Table I. The applicability of the perturbation method enables a restriction of the calculation of the matrix elements of the Hamiltonian in the monoexcited state representation only to the elements $H_{k 1}$, where k denotes the configuration for which the diagonal element $K_{\mathrm{kk}}$ is the smallest between all configurations which we must consider for the transition given. Further, it is necessary to compute all diagonal elements.

Table II

Excitation Energies of the $\beta$-Band

$\varepsilon_{\mathrm{H}}^{\beta}$ according to Hückel approximation in $\beta$ units, $\varepsilon_{\mathrm{P}}^{\beta}$ calculated by first order configuration interaction, $\varepsilon^{\beta}$ obtained by configuration interaction to $1|\beta|, \varepsilon_{\exp }^{\beta}$ experimental values ${ }^{2}\left(20^{\circ} \mathrm{C}\right)$; $\varepsilon_{\mathrm{p}}^{\beta}, \varepsilon,{ }^{\beta} \varepsilon_{\exp }^{\beta}$ are given in $\mathrm{eV}$.

\begin{tabular}{|c|c|c|c|c|c|c|}
\hline Design. & Compound & $\varepsilon_{\mathrm{H}}^{\beta}(\beta)$ & $\varepsilon_{\mathrm{P}}^{\beta}$ & $\varepsilon^{\beta}$ & $\varepsilon_{\exp }^{\beta}$ & $\varepsilon_{\mathrm{exp}}^{\beta}-\varepsilon^{\beta}$ \\
\hline 1 & benzene & $2 \cdot 000$ & $6 \cdot 82$ & $6 \cdot 82$ & 6.77 & -0.05 \\
\hline 2 & naphthalene & 1.618 & $5 \cdot 75$ & $5 \cdot 72$ & $5 \cdot 61$ & $-0 \cdot 11$ \\
\hline 3 & phenanthrene & $1 \cdot 374$ & $5 \cdot 05$ & $4 \cdot 93$ & $4 \cdot 94$ & +0.01 \\
\hline 4 & anthracene & $1 \cdot 414$ & $5 \cdot 09$ & $5 \cdot 04$ & 4.92 & $-0 \cdot 12$ \\
\hline 5 & benzo $[c]$ phenanthrene & $1 \cdot 222$ & $4 \cdot 51$ & $4 \cdot 44$ & $4 \cdot 41$ & -0.03 \\
\hline 6 & pyrene & $1 \cdot 324$ & $4 \cdot 76$ & $4 \cdot 63$ & $4 \cdot 55$ & -0.08 \\
\hline 7 & chrysene & $1 \cdot 312$ & $4 \cdot 91$ & $4 \cdot 88$ & $4 \cdot 65$ & $-0 \cdot 23$ \\
\hline 8 & benzo $[a]$ anthracene & $1 \cdot 167$ & $4 \cdot 43$ & $4 \cdot 31$ & $4 \cdot 28$ & -0.03 \\
\hline 9 & naphthalene & $1 \cdot 295$ & $4 \cdot 76$ & $4 \cdot 71$ & $4 \cdot 53$ & $-0 \cdot 18$ \\
\hline 10 & picene & $1 \cdot 182$ & $4 \cdot 46$ & $4 \cdot 32$ & $4 \cdot 32$ & $+0 \cdot 00$ \\
\hline 11 & perylene & $1 \cdot 347$ & $4 \cdot 87$ & $4 \cdot 80$ & $4 \cdot 96$ & $+0 \cdot 16$ \\
\hline 12 & naphthalene & 0.958 & $3 \cdot 85$ & $3 \cdot 78$ & $3 \cdot 91$ & $+0 \cdot 13$ \\
\hline 13 & benzo[a]pyrene & $1 \cdot 173$ & $4 \cdot 49$ & $4 \cdot 29$ & $4 \cdot 18$ & $-0 \cdot 11$ \\
\hline 14 & dibenzo $[a, c]$ anthracene & $1 \cdot 213$ & $4 \cdot 39$ & $4 \cdot 34$ & $4 \cdot 28$ & -0.06 \\
\hline 15 & dibenzo $[a, h]$ anthracene & $1 \cdot 158$ & $4 \cdot 56$ & $4 \cdot 15$ & $4 \cdot 13$ & -0.02 \\
\hline 16 & pentacene & $1 \cdot 220$ & $4 \cdot 49$ & $4 \cdot 29$ & $4 \cdot 00$ & -0.29 \\
\hline 17 & benzo[e]pyrene & $1 \cdot 215$ & $4 \cdot 47$ & $4 \cdot 43$ & $4 \cdot 29$ & $-0 \cdot 14$ \\
\hline 18 . & benzo $[r s t]$ pentaphene & 1.024 & 3.98 & $3 \cdot 81$ & $3 \cdot 73$ & -0.08 \\
\hline 19 & dibenzo $[b$, def $]$ chrysene $^{a}$ & 1.096 & $4 \cdot 05$ & $4 \cdot 03$ & 3.97 & -0.09 \\
\hline 20 & coronene & 1.078 & $4 \cdot 43$ & $4 \cdot 34$ & $4 \cdot 07$ & $-0 \cdot 27$ \\
\hline
\end{tabular}

${ }^{a}$ Compare Table I; the forbidden transition $A_{g}$ is given. 


\section{Results and Discussion}

Comparison of the Results of Calculation with Experimental Data

Tables II and III and Figs 1 and 2 give the results of calculations of the $\beta$ - and $\alpha$ bands and comparison with the experimental data. The assignment of experimental bands was carried out according to $\mathrm{Clar}^{2}$. The $\beta$-band of benzene was taken according to Mason ${ }^{9}$. The theoretically calculated position of the $\beta$-band in dibenzo[b,def $]$ chrysene according to definition previously given shows worse agreement with the experiment than the forbidden second longest-wavelength transition $A_{\mathrm{g}}$. It cannot be ruled out that the maximum experimentally found assigned to the $\beta$-band may be formed by superposition of the band corresponding to the $A_{g}$ transition on the band due to the permitted $B_{u}$ transition of somewhat shorter wavelength. Therefore we take

\section{Table III}

Excitation Energies of the $\alpha$-Band

$\varepsilon_{\mathrm{H}}^{\alpha}$ According to Hückel approximation in $\beta$ units, $\varepsilon_{\mathrm{P}}^{\alpha}$ calculated by first order configuration interaction, $\varepsilon^{\alpha}$ obtained by configuration interaction to $1|\beta|, \varepsilon_{\exp }^{\alpha}$ experimental values ${ }^{2}\left(20^{\circ} \mathrm{C}\right) ; \varepsilon_{\mathrm{P}}^{\alpha}$, $\varepsilon^{\alpha}, \varepsilon_{\exp }^{\alpha}$ are given in eV.

\begin{tabular}{|c|c|c|c|c|c|c|c|c|}
\hline Design. & Compound & $\varepsilon_{\mathrm{H}}^{\alpha}(\beta)$ & $\varepsilon_{\mathrm{P}}^{\alpha} b$ & $\varepsilon^{\alpha}$ & $\varepsilon_{\mathbf{e x p}}^{\alpha}$ & $\varepsilon_{\mathbf{e x p}}^{\alpha}-\varepsilon^{\alpha a}$ & $\varepsilon_{\exp }^{\beta} / \varepsilon_{\exp }^{\alpha}$ & $\varepsilon^{\beta} / \varepsilon^{\alpha}$ \\
\hline 1 & benzene & $2 \cdot 000$ & $4 \cdot 76$ & $4 \cdot 76$ & $4 \cdot 76$ & 0.00 & $1 \cdot 42$ & $1 \cdot 43$ \\
\hline 2 & naphthalene & $1 \cdot 618$ & $4 \cdot 13$ & $4 \cdot 00$ & $3 \cdot 99$ & -0.01 & $1 \cdot 41$ & $1 \cdot 43$ \\
\hline 3 & phenanthrene & $1 \cdot 374$ & $3 \cdot 98$ & $3 \cdot 77$ & $3 \cdot 60$ & -0.17 & $1 \cdot 37$ & $1 \cdot 31$ \\
\hline 4 & anthracene & $1 \cdot 414$ & $3 \cdot 81$ & $3 \cdot 66$ & - & - & - & $1 \cdot 38$ \\
\hline 5 & benzo $[c]$ phenanthrene & $1 \cdot 222$ & $3 \cdot 87$ & $3 \cdot 65$ & $3 \cdot 33$ & $-0 \cdot 32$ & $1 \cdot 32$ & $1 \cdot 22$ \\
\hline 6 & pyrene & $1 \cdot 324$ & $3 \cdot 59$ & $3 \cdot 43$ & $3 \cdot 34$ & -0.09 & $1 \cdot 36$ & $1 \cdot 35$ \\
\hline 7 & chrysene & $1 \cdot 312$ & $3 \cdot 77$ & $3 \cdot 59$ & $3 \cdot 45$ & $-0 \cdot 14$ & $1 \cdot 35$ & $1 \cdot 36$ \\
\hline 8 & benzo $[a]$ anthracene & $1 \cdot 167$ & $3 \cdot 83$ & $3 \cdot 59$ & $3 \cdot 22$ & $-0 \cdot 37$ & $1 \cdot 33$ & $1 \cdot 20$ \\
\hline 9 & naphthacene & $1 \cdot 295$ & $3 \cdot 75$ & $3 \cdot 59$ & - & -0.37 & - & $1 \cdot 31$ \\
\hline 10 & picene & $1 \cdot 182$ & $3 \cdot 85$ & $3 \cdot 39$ & $3 \cdot 30$ & -0.09 & $1 \cdot 31$ & $1 \cdot 27$ \\
\hline 11 & perylene & $1 \cdot 347$ & $3 \cdot 84$ & $3 \cdot 66$ & 3.67 & 0.01 & $1 \cdot 35$ & $1 \cdot 31$ \\
\hline 12 & pentaphene & 0.958 & $3 \cdot 58$ & $3 \cdot 30$ & $2 \cdot 93$ & -0.37 & $1 \cdot 33$ & $1 \cdot 15$ \\
\hline 13 & benzo $[a]$ pyrene & $1 \cdot 173$ & $3 \cdot 49$ & $3 \cdot 34$ & $3 \cdot 08$ & $-0 \cdot 26$ & $1 \cdot 36$ & $1 \cdot 28$ \\
\hline 14 & dibenzo $[a, c]$ anthracene & $1 \cdot 213$ & $3 \cdot 71$ & $3 \cdot 47$ & $3 \cdot 31$ & $-0 \cdot 16$ & $1 \cdot 29$ & $1 \cdot 25$ \\
\hline 15 & dibenzo $[a, h]$ anthracene & $1 \cdot 158$ & $3 \cdot 54$ & $3 \cdot 37$ & $3 \cdot 14$ & -0.23 & $1 \cdot 32$ & $1 \cdot 23$ \\
\hline 16 & pentacene $^{a}$ & 0.838 & $3 \cdot 41$ & $3 \cdot 10$ & $2 \cdot 90$ & -0.20 & $1 \cdot 38$ & $1 \cdot 38$ \\
\hline 17 & benzo[e]pyrene & $1 \cdot 215$ & $3 \cdot 74$ & $3 \cdot 56$ & $3 \cdot 19$ & -0.37 & $1 \cdot 34$ & $1 \cdot 24$ \\
\hline 18 & benzo $[r s t]$ pentaphene & $1 \cdot 024$ & $3 \cdot 43$ & $3 \cdot 22$ & $2 \cdot 86$ & $-0 \cdot 36$ & $1 \cdot 30$ & $1 \cdot 18$ \\
\hline 19 & dibenzo $[b$, def $]$ chrysene ${ }^{b}$ & $1 \cdot 096$ & $3 \cdot 93$ & $3 \cdot 75$ & - & - & - & $1 \cdot 07$ \\
\hline 20 & coronene & $1 \cdot 078$ & $3 \cdot 19$ & $3 \cdot 17$ & $2 \cdot 90$ & $-0 \cdot 27$ & $1 \cdot 40$ & $1 \cdot 37$ \\
\hline
\end{tabular}

${ }^{a}$ Compare Table I. The transition $B_{1 \mathrm{~g}}$ is given; ${ }^{b}$ Compare Table I. The transition $B_{\mathrm{u}}$ is given. 
in the Tables the excitation energy of the corresponding $A_{g}$ transition even though this assignment is disputable. Figs $3 a-d$ compare the excitation energies calculated with the absorption curves experimentally observed and also show additional tran-

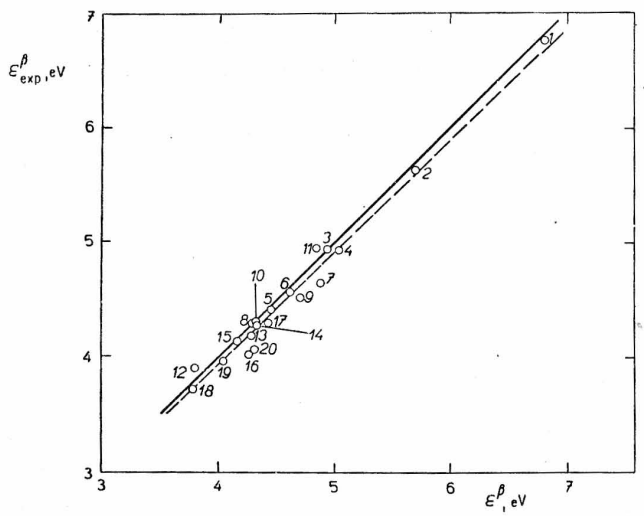

Fig. 1

Comparison of Calculated Excitation Energies $\varepsilon^{\beta}$ with Corresponding Experimental Values $\varepsilon_{\exp }^{\beta}$ Designation of compounds, see Table II. Full straight line $\varepsilon^{\beta}=\varepsilon_{\exp }^{\beta}$; the regression line is dashed.

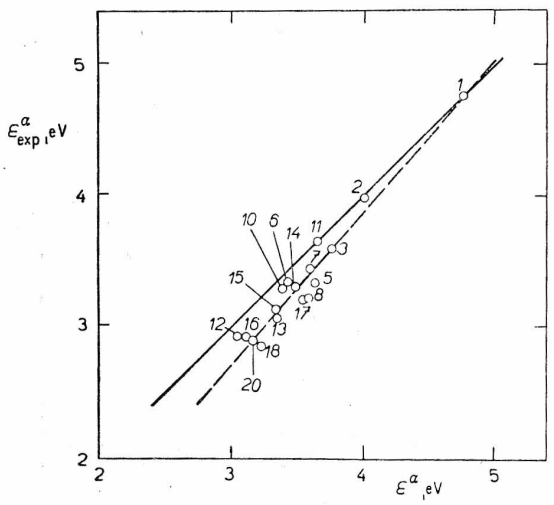

Fig. 2

Comparison of Calculated Excitation Energies $\varepsilon^{\alpha}$ with Corresponding Experimental Values $\varepsilon_{\exp }^{\alpha}$ Designation of compounds see Table III. Full straight line $\varepsilon_{\exp }^{\alpha}=\varepsilon^{\alpha}$, the regression line is dashed. 
sitions belonging to the irreducible representations from Table I which occur in the near ultraviolet region. Only the interaction of the configurations of the same excitation energy was considered in the calculation of these additional transitions. Otherwise stated, the positions of the corresponding maxima belonging to these transitions are estimated from the values of the diagonal elements of the Hamiltonian for the following functions

$$
|1 ; i, k ; \pm\rangle=1 / \sqrt{ } 2\left[\left|1, i \rightarrow k^{\prime}\right\rangle \pm\left|1, k \rightarrow i^{\prime}\right\rangle\right],
$$

and

$$
|1, i . i\rangle=\left|1 ; i \rightarrow i^{\prime}\right\rangle \text {. }
$$

The correlation and regression coefficients of the dependences of the experimental excitation energy on the theoretical estimates $\varepsilon_{\mathrm{H}}^{\mathrm{k}}$, $\varepsilon_{\mathrm{P}}^{\mathrm{k}}$ and $\varepsilon^{\mathrm{k}}(k=\alpha, \beta)$ are given in Table IV. $\beta_{\mathrm{H}}^{\mathrm{k}}$ is the excitation energy according to Hückel (using the value $\beta=$ $=-2 \cdot 318 \mathrm{eV}), \varepsilon^{\mathrm{k}}$ is the excitation energy calculated by configuration interaction up to $1|\beta|$.

The agreement of the $\beta$-band is satisfactory since the standard deviation of the theoretical and experimental excitation energies is only little larger than $0.1 \mathrm{eV}$ $(0.125 \mathrm{eV}$ for the set without pentacene, $0.140 \mathrm{eV}$ for the whole set studied). The agreement for pentacene is similarly as in the case of the $p$-band considerably less

\section{Table IV}

Correlation Coefficients $r$, Regression Coefficients ${ }^{a} s$, Standard Deviations ${ }^{b} \Delta y$ and Mean Values $\bar{y}$ for the Dependence of the Calculated Excitation Energies on the Experimental Values for the $\beta$-Band

\begin{tabular}{|c|c|c|c|c|}
\hline $\begin{array}{c}\text { Quantities } \\
\text { Calculated }\end{array}$ & $\varepsilon_{\mathbf{H}}^{\beta}(\beta)$ & $\varepsilon_{\mathbf{P}}^{\beta}$ & $\varepsilon^{\beta}$ & $\varepsilon_{\exp }^{\beta}$ \\
\hline$r\left(\varepsilon_{\exp }^{\beta}, y\right)$ & 0.977 & 0.980 & 0.987 & - \\
$r^{\prime}\left(\varepsilon_{\exp }^{\beta}, y\right)$ & 0.986 & 0.984 & 0.990 & - \\
$s\left(\varepsilon_{\exp }^{\beta}, y\right)$ & 1.286 & 1.037 & 0.992 & - \\
$s^{\prime}\left(\varepsilon_{\exp }^{\beta}, y\right)$ & 1.280 & 1.029 & 0.985 & - \\
$s\left(y, \varepsilon_{\exp }^{\beta}\right)$ & 0.742 & 0.925 & 0.981 & - \\
$s^{\prime}\left(y, \varepsilon_{\exp }^{\beta}\right)$ & 0.758 & 0.942 & 0.993 & - \\
$\Delta y$ & 1.635 & 0.236 & 0.140 & - \\
$\Delta y^{\prime}$ & 1.660 & 0.214 & 0.125 & - \\
$y$ & 2.941 & 4.076 & 4.603 & 4.523 \\
$y^{\prime}$ & 2.859 & 4.717 & 4.619 & 4.551 \\
\hline
\end{tabular}

${ }^{a}$ The regression line equation: $y-\bar{y}=s(y, x) \cdot(x-\bar{x}){ }^{b} \Delta y=\left\{\sum_{\mathbf{i}}\left(y_{\mathrm{i}}-\varepsilon_{\exp , \mathrm{i}}^{\mathrm{K}}\right) /(N-1)\right\}^{1 / 2}$, where i denotes the compound and $N$ the number of the compounds studied; ${ }^{c}$ the primed quantities refer to the set of hydrocarbons without pentacene. 
Koutecký, Paldus, Vitek:
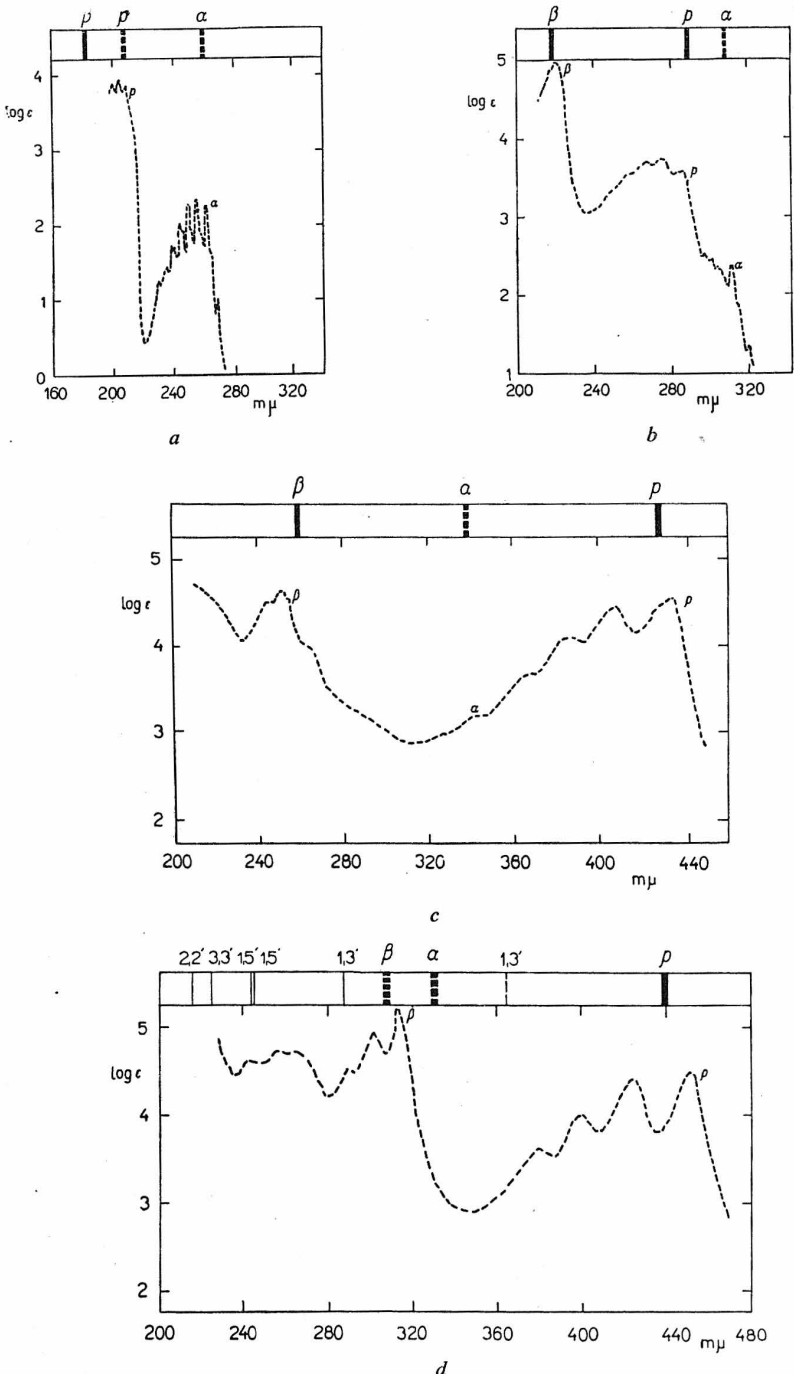
precise than that found for other compounds and again it is evident that linear annelation produces anomalous effects which phenomenon should further be studied. The agreement of theoretical and experimental values is far less satisfactory for the $\alpha$-band (the standard deviation is 0.249 for the set without pentacene and 0.246 for the whole set studied). Clar $^{2}$ has found that the ratio of the wavelengths of the $\alpha$-band and $\beta$-band $\lambda_{\alpha} / \lambda_{\beta}$ is approximately constant and equal to $1 \cdot 3$ (compare ref. ${ }^{9}$ ). Table III also demonstrates that the corresponding theoretical values of this ratio are also found to have approximately this walue even though they are lower than the values experimentally observed for the majority of larger hydrocarbons. However, it is interesting that the larger theoretical values in the ratio $\lambda_{\alpha} / \lambda_{\beta}$ for benzene and naphthalene (higher than 1.4) agree well with the experimental values. The excessive decrease of the theoretical ratio $\lambda_{\alpha} / \lambda_{\beta}$ for larger hydrocarbons is evidently related to the too small values of $\lambda_{\alpha}$ predicted on the basis of the calculations carried out in this study.

Table V

Correlation Coefficients $r$, Regression Coefficients ${ }^{a} s$, Standard Deviations $\Delta y$ and Mean Values $\bar{y}$ for the Dependence of the Calculated Excitation Energies on the Experimental Values for the $\alpha$-Band

\begin{tabular}{|c|c|c|c|c|}
\hline $\begin{array}{c}\text { Quantities } \\
\text { Calculated }^{b}\end{array}$ & $\varepsilon_{\mathbf{H}}^{\alpha}$, & $\varepsilon_{\mathbf{P}}^{\alpha}$ & $\varepsilon^{\alpha}$ & $\varepsilon_{\exp }^{\alpha}$ \\
\hline & & & & \\
$r^{\prime}\left(\varepsilon_{\exp }^{\alpha}, y\right)$ & 0.972 & 0.944 & 0.975 & - \\
$s\left(\varepsilon_{\exp }^{\alpha}, y\right)$ & 0.983 & 0.938 & 0.970 & - \\
$s^{\prime}\left(\varepsilon_{\exp }^{\alpha}, y\right)$ & 0.753 & 1.270 & 1.188 & - \\
$s\left(y, \varepsilon_{\exp }^{\alpha}\right)$ & 0.808 & 1.268 & 1.203 & - \\
$s^{\prime}\left(y, \varepsilon_{\exp }^{\alpha}\right)$ & 1.254 & 0.701 & 0.800 & - \\
$\Delta y$ & 1.196 & 0.695 & 0.781 & - \\
$\Delta y^{\prime}$ & 0.501 & 0.448 & 0.246 & - \\
$\bar{y}^{\prime}$ & 0.464 & 0.443 & 0.249 & - \\
& 2.890 & 3.748 & 3.551 & 3.351 \\
& 3.315 & 3.769 & 3.579 & 3.379 \\
\hline
\end{tabular}

${ }^{a}$ For symbols see Table IV; ${ }^{b}$ the primed quantities refer to the set of hydrocarbons without pentacene.

Fig. 3

Comparison of Experimental Electronic Spectrum with Calculated Excitation Energies $a$ Benzene, $b$ naphthalene, $c$ perylene, $d$ dibenzo[b,def $]$ chrysene. The permitted transitions are full, forbidden transitions are dashed. The estimates of positions of the $p-, \alpha-$, and $\beta$-bands corresponding to the excitation energies are depicted above the spectra. Other excitation energies were estimated on the basis of the values of the corresponding diagonal elements of $H$ in the representation of functions of the type (2). 
The deviations of the $\alpha$-band are not caused by insufficient extent of the configuration interaction. Table VI which gives the results of the extending of the configuration interaction for the representatives of various symmetry groups demonstrates that such extending has, however, little effect on the results of calculations.

\section{Interpolation Formula for Individual Absorption Bands}

The Hamiltonian matrix elements in the monoexcited state representation used in our approximation are dependent on the quantities of the three types: on the resonance integral $\beta$, on the integrals $\gamma_{\mu \mu}=I-A^{3}$ and integrals $\gamma_{\mu v}(\mu \neq v)$ taken equal to $\gamma_{\mu \mu}$ for $r \rightarrow 0$. At the same time it is necessary to take the integrals $\gamma_{\mu \mu}$ as quantities of different physical type than $\gamma_{\mu \nu}$ for $\mu \neq v$. It may occur that the considerably simplified method using only two semiempirical parameters artificially reduced quantities of different types into a single mathematical expression. It is interesting that the matrix elements of the type

$$
\left\langle 1 ; i, k ;-\left|\mathrm{H}-E_{0}\right| ; i, k ;-\right\rangle,
$$

where $E_{0}$ is the ground state energy, are independent on the values of $\gamma_{\mu \mu}$, and therefore the position of the $\alpha$-band is not dependent on the values of $\gamma_{\mu \mu}$.

\section{Table VI}

Influence of the Extent of Configuration Interaction

$\varepsilon^{k}$ Excitation energy for CI to $1|\beta|$. $\varepsilon_{\mathrm{c}}^{\mathrm{k}}$ excitation energy for CI extended by the number of configurations given in the Table in contrast to the original limitation.

\begin{tabular}{|c|c|c|c|c|c|c|c|c|c|}
\hline $\begin{array}{l}\text { De- } \\
\text { sign. }\end{array}$ & Compound & $\begin{array}{c}\text { Original } \\
\text { Number of } \\
\text { Configurat- } \\
\text { ions }\end{array}$ & $\begin{array}{c}\text { Extended } \\
\text { Number of } \\
\text { Configurat- } \\
\text { ions }\end{array}$ & $\varepsilon^{\beta}$ & $\varepsilon_{\mathrm{c}}^{\beta}$ & $\varepsilon^{\beta}-\varepsilon_{\mathbf{c}}^{\beta}$ & $\varepsilon^{\alpha}$ & $\varepsilon_{\mathrm{c}}^{\alpha}$ & $\varepsilon^{\alpha}-\varepsilon_{\mathbf{c}}^{\alpha}$ \\
\hline 4 & anthracene & 5 & 6 & $5 \cdot 04$ & $5 \cdot 04$ & 0.00 & 3.66 & 3.64 & 0.02 \\
\hline 7 & chrysene & 7 & 24 & $4 \cdot 88$ & $4 \cdot 81$ & 0.07 & 3.59 & $3 \cdot 53$ & 0.06 \\
\hline 8 & benzo $[a]$ anthracene & 13 & 45 & $4 \cdot 31$ & $4 \cdot 29$ & 0.02 & $\cdot 3 \cdot 59$ & $3 \cdot 57$ & 0.02 \\
\hline 17 & benzo $[e]$ pyrene & 7 & 12 & $4 \cdot 43$ & $4 \cdot 39$ & $0 \cdot 04$ & $3 \cdot 56$ & $3 \cdot 53$ & 0.03 \\
\hline 20 & coronene & 4 & 7 & $4 \cdot 34$ & $4 \cdot 32$ & 0.02 & $3 \cdot 17$ & 3.03 & $0 \cdot 14$ \\
\hline
\end{tabular}

In view of many simplifications in the method employed one cannot rule out that for different bands quantities of different type or different magnitude are neglected or that the positions of some bands are influenced by quantities which show no similar effect for other bands. For different excited states different SCF LCAO molecular orbitals should in fact be employed. Since we use the Hückel molecular orbitals we must expect that individual semiempirical parameters are different for various bands ${ }^{10}$. Therefore one arrives at the conclusion to choose different empirical constants for different bands (compare refs ${ }^{10,11}$ ). However, it should be emphasized 
that the expressions thus obtained for the positions of various absorption bands have only the character of interpolation formulas dependent on the theory employed. For this reason it is economical to use as a basis the simple empirical formulas and to vary the semiempirical parameters in order to obtain the best agreement with experiment. The usefulness of these expressions may only be confirmed when used for the collection of compounds which is satisfactorily representative. We assume our set of twenty hydrocarbons to be considered as representative.

We employed the following interpolation form ulas for alternating benzenoid hydrocarbons

$$
\varepsilon_{\mathrm{a}}^{\mathrm{k}}=\beta^{\mathrm{k}} \varepsilon_{\mathrm{H}}^{\mathrm{k}}+\gamma_{11}^{\mathrm{k}} F^{\mathrm{k}}+\kappa^{\mathrm{k}} G^{\mathrm{k}}, \quad k=p, \alpha, \beta,
$$

where $\beta^{\mathrm{k}}, \gamma_{11}^{\mathrm{k}}, \kappa^{\mathrm{k}}$ are the semiempirical parameters and $\varepsilon_{\mathrm{H}}^{\mathrm{k}}$ the excitation energy according to Hückel in $\beta$ units. $F_{\mathrm{P}}^{\mathrm{k}}$ is the expression appearing in $\varepsilon_{\mathrm{P}}^{\mathrm{k}}$ at $\gamma_{11}^{\mathrm{k}}$

$$
F^{\mathrm{k}}=2^{\delta_{\mathrm{k}, \beta}}\left(1-\delta_{\mathrm{k}, \alpha}\right) \sum_{\mu} c_{1 \mu}^{2} c_{\mathrm{n}^{\mathrm{k}} \mu}^{2},
$$

where $c_{k, \mu}$ is the coefficient for the $\mu$ th atomic orbital in the $k$ th molecular orbital. $G^{\mathrm{k}}$ denotes the part of $\varepsilon_{\mathrm{P}}^{\mathrm{k}}$ dependent on $\gamma_{\mu v}(\mu \neq v)$. For these quantities we find

$$
\begin{gathered}
G^{\mathrm{k}}=\sum_{\mathrm{i}=1}^{\mathrm{N} / 2}\left[\left(\begin{array}{ll}
1 & i \\
1 & i
\end{array}\right)+\left(\begin{array}{ll}
n^{\mathrm{k}} & i \\
n^{\mathrm{k}} & i
\end{array}\right)\right]-\left(\begin{array}{cc}
1 & 1 \\
n^{\mathrm{k}} & n^{\mathrm{k}}
\end{array}\right)+ \\
+(-1)^{\delta_{\mathrm{k}, \beta}}\left(1-\delta_{\mathrm{k}, \mathrm{P}}\right)\left(\begin{array}{ll}
1 & n^{\mathrm{k}} \\
1^{\prime} & n^{\mathrm{k}^{\prime}}
\end{array}\right)+2(2)^{\delta_{\mathrm{k}, \beta}}\left(1-\delta_{\mathrm{k}, \alpha}\right)\left(\begin{array}{ll}
1 & n^{\mathrm{k}^{\prime}} \\
1 & n^{\mathrm{k}^{\prime}}
\end{array}\right) \\
k=p, \alpha, \beta,
\end{gathered}
$$

where

$$
\left(\begin{array}{l}
i j \\
l m
\end{array}\right)=\sum_{\mu \neq v} c_{\mathrm{i} \mu} c_{\mathrm{j} \mu} c_{\mathrm{lv}} c_{\mathrm{mv}} \gamma_{\mu \mathrm{v}},
$$

and $\gamma_{\mu v}$ are defined by equation $(1) \cdot n^{\mathrm{p}}=1, n^{\beta}$ is the number of the molecular orbital whose element

$$
\left\langle 1 ; 1, n^{\beta} ;+\left|\mathrm{H}-E_{0}\right| 1 ; 1, n^{\beta} ;+\right\rangle
$$

has the smallest absolute value $\left(n^{\beta} \neq 1\right)$ and the corresponding transition is permitted. Compound 19 is an exception in our assignment as we take $n^{\beta}=2$ although the corresponding transition is forbidden. Analogously $n^{\beta}$ is the number of the molecular orbital whose element

$$
\left\langle 1 ; 1, n^{\alpha} ;-\left|H-E_{0}\right| 1 ; 1, n^{\alpha} ;-\right\rangle
$$

is the smallest and the corresponding transition is forbidden. The expressions for $F^{\mathrm{k}}$ and $G^{\mathrm{k}}$, given in equations (5) and (6), are valid only for those compounds whose highest and second highest filled orbitals in the ground state are nondegenerate due to symmetry. For these compounds (in our case for benzene and coronene) it is necessary to consider the configuration interaction to such extent to include configurations formed by transitions of electrons between these orbitals and the corresponding 
virtual orbitals. The parameter $\left(\kappa^{\mathbf{k}}\right)^{-1}$ may be interpreted as a "dielectric constant" (compare the study performed by $\mathrm{Julg}^{12}$ ).

The parameters $\beta^{\mathrm{k}}, \gamma_{11}^{\mathrm{k}}, \kappa^{\mathrm{k}}$ obtained by minimization of the sum of the squares of deviations $\left(\varepsilon_{\exp }^{\mathrm{k}}-\varepsilon_{\mathrm{a}}^{\mathrm{k}}\right)$ for the set of nineteen compounds (without pentacene) are given in Table VI, where the standard deviations are also included. The values of these deviations together with Fig. 4 demonstrate that the interpolation formulas describe very well the positions of the $\alpha-, \beta$-, and $p$-bands experimentally observed. Pentacene again shows an exceptional behaviour.

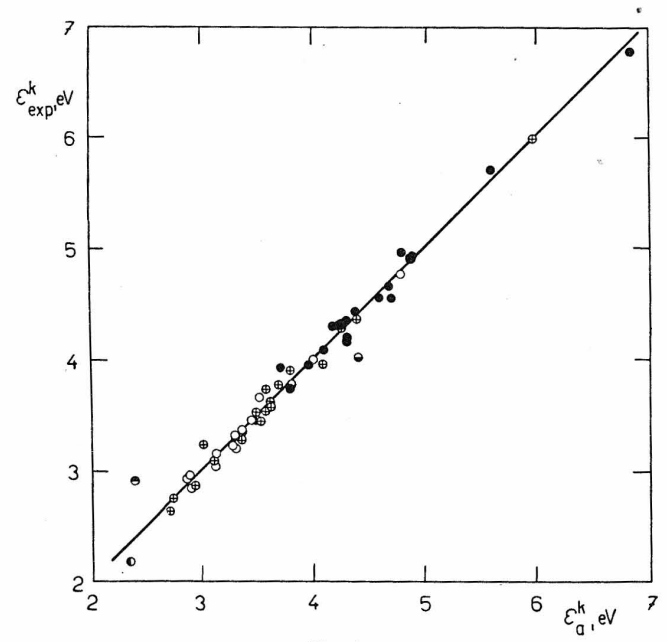

Fig. 4

Comparison of the Excitation Energies $\varepsilon_{\mathrm{a}}^{\mathrm{k}}$ Calculated from Equation (4) with the Corresponding Experimental Values $\varepsilon_{\exp }^{\mathbf{k}}$

$\bigcirc \alpha$-band; $\beta$-band, $\oplus p$-band; $\ominus \alpha$-band of pentacene, $\ominus \beta$-band of pentacene, $\odot p$-band of pentacene.

\section{Comparison with the Hückel Method}

Fig. 5 shows that the correlation of the positions of the $\alpha$ - and $\beta$-bands experimentally observed with the corresponding Hückel excitation energies $\varepsilon_{\mathrm{H}}$ is no doubt less precise than that found for the $p$-band but it is quite good even in this case (compare Table IV). It is characteristic that the regression line for the $\alpha$-band in Fig. 5 lies below the regression line for the $\beta$-band and has a considerably smaller slope $(0.753$ in contrast to 1.286$)$. Fig. 6 , where the theoretical values $\varepsilon^{\mathrm{k}}(k=\alpha, \beta)$ are plotted against $\varepsilon_{\mathrm{H}}^{\mathrm{k}}(k=\alpha, \beta)$, shows a considerable resemblance to Fig. 5 . 
Table VII

Semiempirical Parameters $\beta^{\mathbf{k}}, x^{\mathbf{k}}$, and $\gamma_{11}^{\mathbf{k}}$ (Equation (4)) and Standard Deviations ${ }^{a} \Delta y^{\mathbf{k}}$

\begin{tabular}{|c|c|c|c|c|c|}
\hline $\begin{array}{c}\text { Relevant } \\
\text { Quantity }\end{array}$ & $\beta^{\mathbf{k}}$ & $x^{\mathbf{k}}$ & $\gamma_{11}^{\mathbf{k}}$ & $\Delta y^{\mathbf{k}}$ & $\Delta y^{\mathbf{k}}$ \\
\hline & 2.392 & 0.876 & 9.517 & 0.088 & 0.078 \\
$\beta$ & 2.484 & 0.678 & 9.713 & 0.133 & 0.096 \\
$\alpha$ & 2.363 & 0.454 & - & 0.144 & 0.056 \\
\hline
\end{tabular}

${ }^{a}$ In this case the standard deviation is defined $\Delta y=\left\{\underset{\mathbf{i}}{\sum}\left[\left(\varepsilon_{\mathrm{a}}^{\mathbf{k}}\right)_{\mathbf{i}}-\varepsilon_{\exp , \mathbf{i}}^{\mathrm{k}}\right] /(N-1)\right\}^{1 / 2}$; where $\varepsilon_{\mathrm{a}}^{\mathrm{k}}$ is the excitation energy calculated for the ith compound and kth band according to equation (4), $\varepsilon_{\exp }^{\mathrm{k}}$ is the corresponding experimental value, $N$ is the number of compounds. The primed quantity refers to the hydrocarbon set without pentacene.

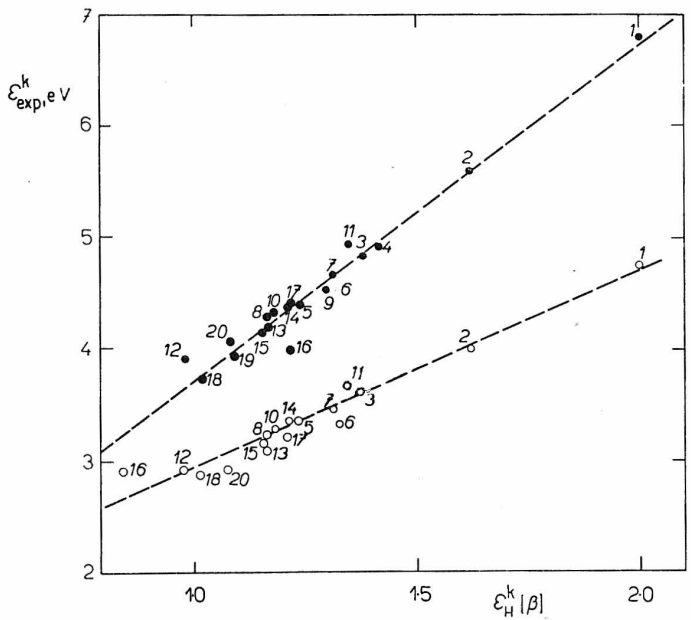

Fig. 5

Dependence of the Experimental Excitation Energies $\varepsilon_{\exp }^{\mathbf{k}}$ on $\varepsilon_{\mathrm{H}}^{\mathbf{k}}$

- $k=\beta, \circ k=\alpha$. For numbering of compounds see Table II. The regression lines are dashed.

For the $p$-band it was found that the part of the expression for the excitation energy which is dependent on the electronic repulsion integrals

$$
\varepsilon^{\mathrm{k}}-\varepsilon_{\mathrm{H}}^{\mathrm{k}}=\Gamma^{\mathrm{k}}
$$




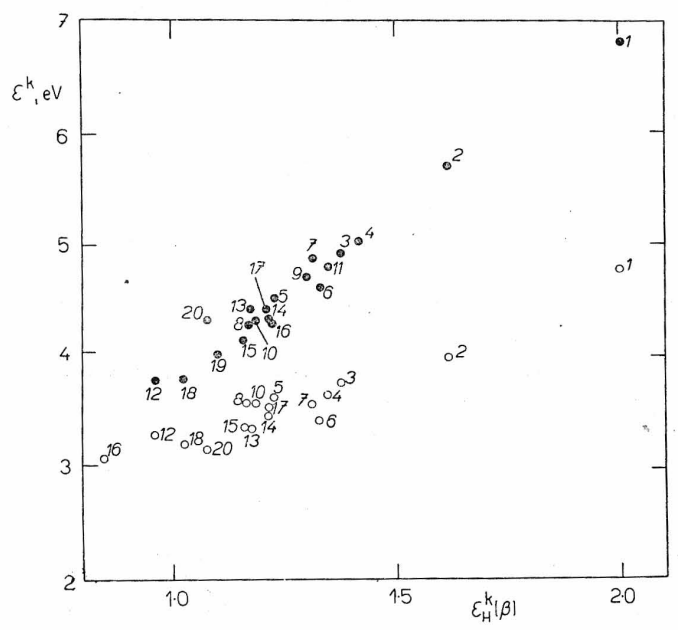

Fig. 6

Dependence of the Theoretical Excitation Energies $\varepsilon^{k}$ on $\varepsilon_{\mathbf{H}}^{k}$

- $k=\beta, \circ k=\alpha$. For numbering of compounds see Table II.

is approximately constant. The analogous quantities for the $\alpha$ - and $\beta$-bands change markedly so that $\Gamma^{\alpha}$ is small for those hydrocarbons for which $\Gamma^{\beta}$ is large and inversely (compare Fig. 7). From Fig. 7 it is also evident that $\Gamma^{\mathrm{k}}$ correlate with the "experimental quantity"

$$
\Gamma_{\exp }^{\mathrm{k}}=\varepsilon_{\exp }^{\mathrm{k}}-\varepsilon_{\mathrm{H}}^{\mathrm{k}} .
$$

On the whole it is evident that the theory considering the interaction of the electrons describes a number of characteristic properties of the $p-, \beta$ - and $\alpha$-bands which cannot be accounted for by the simple MO LCAO method:

1. We have already mentioned that the part of the excitation energy dependent on the electronic repulsion integrals $\Gamma^{\mathrm{P}}$ is approximately constant. Therefore the resonance integral $\beta$ obtained in the Hückel method and that in the method presented show nearly equal values. The mean value of $\Gamma^{\mathrm{p}}$ is $1.39 \mathrm{eV}$ so that the additive shift produced by the terms dependent on electronic repulsion is considerable. Thus it seems that the additive constant $D^{\mathrm{k}}$ in the relations

$$
\varepsilon_{\exp }^{\mathrm{k}}=\beta^{\mathrm{k}} \varepsilon_{\mathrm{H}}^{\mathrm{k}}+D^{\mathrm{k}}
$$

for $k=p$ is related to electronic repulsion $\left(D^{\mathrm{p}}=1 \cdot 32 \mathrm{eV}\right)$. 
2. $\Gamma^{\alpha}$ approximately diminishes with the increasing $\varepsilon_{\mathrm{H}}^{\alpha}$ causing a decrease of the slope of the dependence of $\varepsilon_{\exp }^{\alpha}$ on $\varepsilon_{\mathrm{H}}^{\alpha}$ in contrast to the analogous quantity for the $p$-band. The additive constant appearing in equation (10) is also not negligible for $k=\alpha$ and equals $1 \cdot 18 \mathrm{eV}$.

3. $\Gamma^{\beta}$ approximatelyincreases withincreasing $\varepsilon_{\mathrm{H}}^{\beta}$ so that the slope of the dependence of $\varepsilon_{\exp }^{\beta}$ on $\varepsilon_{\mathrm{H}}^{\beta}$ is larger than the analogous slope for the $p$-band. The additive constant $D^{\beta}$ in equations $(10)$ is equal to $0.82 \mathrm{eV}$.

According to the assumptions made in the theory of Goodman and Shull ${ }^{11}$ the following relation should be valid

$$
\varepsilon^{\alpha}+\varepsilon^{\beta}=\beta^{\prime} \cdot \varepsilon_{\mathrm{H}},
$$

and thus also

$$
\Gamma^{\alpha}+\Gamma^{\beta}=\beta^{\prime \prime} \cdot \varepsilon_{\mathrm{H}} .
$$

The properties given of the $\alpha$-, and $\beta$-bands favour rather the following equation

$$
\Gamma^{\alpha}+\Gamma^{\beta}=d \cdot \varepsilon_{\mathrm{H}}+C,
$$

where $C$ is about $1 \mathrm{eV}$ and the quantity $d$ is very small.

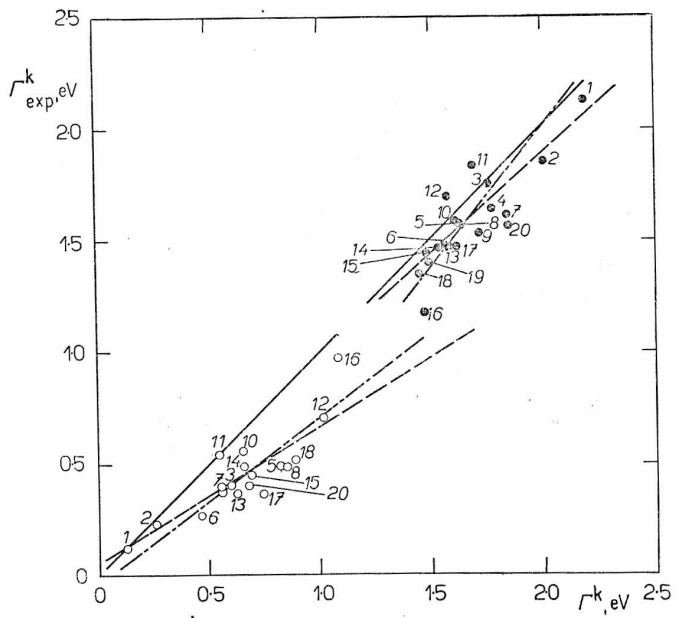

Fig. 7

Dependence of $\Gamma_{\exp }^{\mathrm{k}}$ on $\Gamma^{\mathrm{k}}$

Compare equations $\left(8^{\prime}\right)$ and $(9) . \quad k=\beta, \circ k=\alpha$. 
Our thanks are due to the Research Institute of Organic Syntheses, Pardubice-Rybitvi, for support and interest and to the Technical-Economical Research Institute of the Chemical Industry, Prague, for computing time on the electronic computer Ural I.

\section{References}

1. Koutecký J., Paldus J., Zahradník R.: J. Chem. Phys. 36, 3129 (1962).

2. Clar E.: Aromatische Kohlenwasserstoffe. Springer, Berlin 1952.

3. Pariser R., Parr R.: J. Chem. Phys. 21, 466, 767 (1953).

4. Pariser R.: J. Chem. Phys. 24, 250 (1956).

4. Pariser R.: J. Chem. Phys. 24, 250 (1956).

5. Pople J. A.: Trans. Faraday Soc. 49, 1375 (1953).

6. Pople J. A.: Proc. Phys. Soc. (London) A 68, 81 (1955).

7. Mataga N., Nishimoto K.: Z. physik. Chem. (Frankfurt) 13, 140 (1957).

8. Dewar M. J. S., Longuet-Higgins H. C.: Proc. Phys. Soc. (London) 67, 795 (1954).

9. Mason S. F.: Quart. Revs (London) 15, 287 (1961).

10. Lykos P. G.: J. Chem. Phys. 35, 1249 (1961).

11. Goodman L., Shull H.: J. Chem. Phys. 23, 33 (1955).

12. Julg A.: Tetrahedron, in the press.

13. Friedel R. A., Orchin M.: Ultraviolet Spectra of Aromatic Compounds. Wiley, New York 1951. Translated by J. Jonáš.

\section{Резюме}

Я. Коутецки, И. Палдус и В. Витек: Расчет положений $\alpha-и \beta$-полос в электронном спектре бензоидных углеводородов при помощи метода ограниченного взаимодействия конфигураций. Рассчитаны положения $\alpha$ - и $\beta$-полос электронных спектров двадцати ароматических бензоидньх углеводородов при помощи полуэмпирического метода ограниченного взаимодействия конфигураций в $\pi$-электронном приближении с применением молекулярных орбит Хюккеля. Для $\beta$-полосы установлено хорошее совпадение с экспериментальньми значениями, в то время как в случае $\alpha$-полосы отмечено систематическое отклонение. Это отклонение нельзя устранить расширением взаимодействия конфигураций моновозбуженных состояний, конструированных на основании данных молекулярных орбит. Однако, учтение отталкивания электронов позволяет объяснить характер зависимостей экспериментальных энергий возбуждения от энергий возбуждения на основании простого метода молекулярных орбит Хюккеля. При применении полуэмпирических параметров, различных для электронных переходов (однако не очень отличающихся друг от друга), были получены полуэмпирические интерполяционные формулы для $p$-, $\alpha$-и $\beta$-полос, в случае исследовавшейся серии веществ дающие очень хорошее совпадение с соответствующими экспериментальными энергиями возбуждения. 